\title{
Antimicrobial properties and interaction of two Trp-substituted cationic antimicrobial peptides with a lipid bilayer
}

\author{
Xiaonan $\mathrm{Bi}^{1}$, Che Wang ${ }^{2}$, Weibing Dong ${ }^{1}$, Wei $\mathrm{Zhu}^{1}$ and Dejing Shang ${ }^{1,2}$
}

Tryptophan (Trp) residues reportedly exhibit a strong membrane-disruptive activity, and this property endows Trp-containing antimicrobial peptides (AMPs) with a unique ability to interact with the surface of bacterial cell membranes, possibly improving antimicrobial properties. In this study, we investigated the influence of Trp residues engineered to have a distinct preference for the interface region of lipid bilayers on antimicrobial activity. We designed two Trp-substituted AMPs (I1WL5W and I4WL5W) by replacing Ile or Leu residues with two Trp residues at different positions in the L-K6 peptide, and determined their antimicrobial activity and mechanism of membrane action. Both I1WL5W and I4WL5W exhibited significantly higher antimicrobial activity and lower cytotoxicity against Gram-negative and Gram-positive bacteria compared with L-K6. The Trp-substituted peptides had a disordered structure in aqueous solution and adopted an $\alpha$-helical structure in solutions of $50 \%$ trifluoroethanol/water and $30 \mathrm{~mm}$ SDS. I1WL5W and I4WL5W caused a significant leakage of calcein from liposomes containing membranes that mimicked those of Escherichia coli and Staphylococcus aureus. Scanning electron microscopy analysis suggested that I1WL5W and I4WL5W killed bacteria by disrupting bacterial cell membranes. Furthermore, fluorescence and quenching data from a variety of liposomes, which mimic different cell membranes, indicated that the Trp-substituted peptides could insert into the lipid bilayers and induce blue shifts in the emission spectra of the Trp residues. I1WL5W and 14WL5W were also less susceptible to acrylamide or KI quenchers. The current work may be important for designing novel Trp-containing peptides exhibiting strong antimicrobial abilities by penetrating bacterial membranes.

The Journal of Antibiotics (2014) 67, 361-368; doi:10.1038/ja.2014.4; published online 5 February 2014

Keywords: fluorescence; helicity; interfacial activity; membrane; trp residues

\section{INTRODUCTION}

Antimicrobial peptides (AMPs) are important components of innate immunity in many organisms, including amphibians and mammals, and they can be used as potent weapons against harmful microorganisms. ${ }^{1}$ AMPs interact with bacterial cell membranes and enact their antimicrobial activity by membrane perturbation. Bacterial resistance requires microbes to alter their membrane-lipid composition, an alteration that does not affect AMPs, making them impervious to bacterial resistance. Consequently, AMPs are attractive therapeutic candidates for protection against infectious microbes, including multidrug-resistant bacteria. $^{2-4}$

Native AMPs isolated from the frog skin exhibit a broad spectrum of activities against bacteria and fungi, and they also exhibit obvious cytotoxicity toward normal human cells. Therefore, synthetic analogs with improved antimicrobial activities and low cytotoxicity have been developed that are highly effective against bacterial cells. $^{5-7}$ Most AMPs share certain features, including cationicity, hydrophobicity and amphipathicity, that are essential for disruption of bacterial membranes. ${ }^{8-10}$ In addition, specific amino acids can improve antimicrobial activity. Tryptophan residues have the unique ability to interact with the interface region of a membrane, allowing the peptide to anchor to the bilayer surface. ${ }^{11}$ This behavior has been observed in many tryptophan (Trp)-containing AMPs. For example, a molecular dynamics simulation showed the insertion of the N-terminal region of the short peptide, dermaseptin S3, into the bilayer with the Trp residue penetrating the interface layer of the membrane. ${ }^{12}$ Other simulations have highlighted the importance of Trp residues in peptide-membrane interactions. For example, simulations of pentapeptides demonstrated that Trp residues could associate with the positively charged choline headgroups of the lipid bilayer. ${ }^{13}$ Moreover, the large, bulky, paddle-like shape of the indole sidechain disrupted the highly favorable and cohesive hydrophobic interactions of the lipid acyl chains when it inserted into the hydrocarbon core of a lipid bilayer, leading to high entropic energy costs. ${ }^{14}$ Thus, it has been proposed that the complex electrostatic nature of the interface region of lipid bilayers is ideal for accommodating the equally complex nature of $\operatorname{Trp}$ residues. ${ }^{14}$ 
In a previous study, we investigated the role of Trp residues by designing a series of Trp-substituted peptides of L-K6, a peptide derived from temporin-1CEb obtained from the skin secretions of Chinese brown frog (Rana chensinensis), which is typically localized to woodland and wet lowland meadows in northeastern China. The results showed that the addition of Trp residues increased the antimicrobial activity of AMPs. Trp residues at position 1 or 4 rather than at positions 5, 11 and 12 demonstrated antimicrobial activities against Gram-negative bacteria, whereas Trp residues at position 5 (L5W) demonstrated antimicrobial activities against Gram-positive bacteria. ${ }^{15}$ To further improve the antimicrobial activities of peptides against both Gram-negative and Gram-positive bacteria and to characterize the interaction of Trp-substituted AMPs with the lipid bilayer, we designed two Trp-substituted AMPs (I1WL5W and I4WL5W) by replacing Ile or Leu residues with two Trp residues at different positions in L-K6. We then determined their antimicrobial and hemolytic activities as well as their mechanism of membrane action. Our results clearly showed that I1WL5W and I4WL5W had significant activity against both Gram-negative and Gram-positive bacteria. In addition, each peptide could interact with negatively charged bacterial membranes, but they did not show strong activity toward zwitterionic eukaryotic membranes.

\section{MATERIALS AND METHODS}

\section{Bacterial strains}

Escherichia coli (AS 1.349), Pseudomonas aeruginosa (CGMCC 1.860), Klebsiella pneumoniae subspecies Pneumoniae (CGMCC 1.176), Enterobacter cloacae (CGMCC 1.58), Staphylococcus aureus (AS 1.72), Enterococcus faecalis (CGMCC 1.595), Enterococcus faecium (CGMCC 1.2334) and Bacillus cereus (AS 1.126) were obtained from the China General Microbiological Culture Collection Center (Beijing, China). The Proteus mirabilis (CICC 22931) and Staphylococcus epidermidis (CICC 23664) bacterial strains were acquired from the China Center for Industrial Culture Collection.

\section{Peptide synthesis and purification}

The I1WL5W (WKKIWSKIKKLLK-NH ${ }_{2}$ ) and I4WL5W (IKKWWSKIKKLLK$\mathrm{NH}_{2}$ ) peptides were synthesized in a crude form using the standard Fmoc solid-phase peptide synthesis protocols of GL Biochemistry (Shanghai, China). The peptides were purified to near homogeneity ( $>95 \%$ ) using reverse-phase HPLC on a Vydac $218 \mathrm{TP} 1022 \mathrm{C}-18$ column $(2.2 \mathrm{~cm} \times 25 \mathrm{~cm}$; Separations Group, Hesperia, CA, USA) equilibrated with aqueous acetonitrile/water/ trifluoroacetic acid. The molecular masses of the peptides were confirmed using a matrix-assisted laser desorption ionization mass spectrometer (Shimadzu, Kyoto, Japan).

\section{Antimicrobial activity \\ The antimicrobial activities of the peptides against Gram-negative and Gram- positive bacterial strains were evaluated using the standard microdilution method on 96-well microtiter plates. ${ }^{16}$ Aliquots of culture medium containing bacterial suspensions in mid-logarithmic phase at a concentration of $2 \times 10^{5} \mathrm{CFU} \mathrm{ml}^{-1}$ were added to each well of 96-well microtiter plates containing peptide solution serially diluted to concentrations between 0.39 and $100 \mu \mathrm{m}$. Growth inhibition was evaluated by measuring the absorbance at $600 \mathrm{~nm}$ using a microtiter plate reader after $24 \mathrm{~h}$ of incubation at $37^{\circ} \mathrm{C}$. The MIC was defined as the minimal peptide concentration that completely inhibited bacterial growth. All MIC measurements were the average of four independent experiments.}

\section{Hemolytic activity}

The hemolytic activities of the peptides were evaluated in human red blood cells using a previously reported method. ${ }^{17}$ Fresh human red blood cells were washed three times by centrifugation at $800 \mathrm{~g}$ for $10 \mathrm{~min}$ in $0.9 \%(\mathrm{w} / \mathrm{v}) \mathrm{NaCl}$ and incubated with peptides $\left(100-500 \mu \mathrm{m}\right.$ final concentration) at $37^{\circ} \mathrm{C}$ for $1 \mathrm{~h}$.
After centrifugation, the OD540 was measured for each resuspended pellet solution. In addition, control samples for 0 and $100 \%$ hemolysis were incubated with $0.9 \%(\mathrm{w} / \mathrm{v}) \mathrm{NaCl}\left(A_{\text {blank }}\right)$ or water $\left(A_{\text {water }}\right)$, respectively. Percent hemolysis was calculated according to the following eq: Hemolysis $(\%)=\left(\left(A_{\text {sample }}-A_{\text {blank }}\right) /\left(A_{\text {water }}-A_{\text {blank }}\right)\right) \times 100$. Each measurement was taken in triplicate. ${ }^{18}$ The $\mathrm{HC}_{50}$ was defined as the mean peptide concentration that produced $50 \%$ hemolysis.

\section{Kinetic studies}

The kinetics of the antimicrobial activity of the peptides was evaluated using E. coli (AS 1.349) and S. aureus (AS 1.72). At mid-logarithmic growth phase, bacteria $\left(2 \times 10^{5} \mathrm{CFU} \mathrm{ml}^{-1}\right)$ were incubated by shaking at $37^{\circ} \mathrm{C}$ for 0-180 min after peptides were added at final concentrations of $1 \times, 2 \times, 4 \times$ and $8 \times$ the MIC. Aliquots were taken at the indicated times, appropriately diluted and plated on LB agar plates. The CFUs were counted after $16 \mathrm{~h}$ of incubation at $37^{\circ} \mathrm{C}$. All measurements were performed in triplicate.

\section{CD spectroscopy}

The mean residue molar ellipticities of I1WL5W and I4WL5W were determined by CD spectroscopy using a J-810 spectropolarimeter (JASCO, Victoria, British Columbia, Canada). The spectra were measured between 190 and $250 \mathrm{~nm}$ at $0.1 \mathrm{~nm}$ intervals at $25^{\circ} \mathrm{C}$ with a scan rate of $20 \mathrm{~nm} \mathrm{~min}^{-1}$. Peptides at a constant concentration of $0.3 \mathrm{mg} \mathrm{ml}^{-1}$ were dissolved in three different solvents (water, $30 \mathrm{~mm}$ SDS and $50 \%(\mathrm{v} / \mathrm{v})$ trifluoroethanol (TFE)/water), and a quartz cuvette with a 1-mm path length was used in the measurements. The spectra from three consecutive scans were averaged, and the CD spectrum for the appropriate solvent was subtracted from each corresponding peptide spectrum. The percentage of helicity was calculated using the CDNN program.

\section{Preparation of liposomes}

Phosphatidylcholine (PC), phosphatidylethanolamine (PE), phosphatidylglycerol (PG), cholesterol and cardiolipin (CL) were purchased from Sigma (Shanghai, China). Liposomes were prepared by sonication of the required amount of PG/PE/CL $(2: 7: 1, \mathrm{w} / \mathrm{w} / \mathrm{w}), \mathrm{PG} / \mathrm{CL}(3: 1, \mathrm{w} / \mathrm{w})$ or PC/cholesterol $(10: 1, \mathrm{w} / \mathrm{w})$, mimic the membrane of the E. coli type, the $S$. aureus type and the human erythrocyte type, respectively. ${ }^{19-21}$ The phospholipids were dissolved in chloroform at each of the previously described ratios and dried, and the solvents were removed via rotary evaporation to form a multilamellar liposome. Any remaining trace amounts of organic solvent were then completely removed by lyophilization overnight, and the dry lipid film was resuspended in 2-3 $\mathrm{ml}$ of $5 \mathrm{~mm} \mathrm{~N}$-Tris (hydroxymethyl) methyl-2-aminoethanesulfonic acid (TES) buffer ( $\mathrm{pH}$ 7.4) containing $0.1 \mathrm{M} \mathrm{NaCl}$ with gentle vortexing. The suspension was sonicated at $40{ }^{\circ} \mathrm{C}$ for $8 \mathrm{~min}$ using a probe-type sonicator. The liposomes were immediately used for titration measurements.

Liposomes with encapsulated calcein were prepared using the freeze-thaw method. ${ }^{18,20,22}$ Briefly, the phospholipids were dissolved in chloroform at each of the described ratios. After vacuum evaporation and overnight drying, a dye solution ( $90 \mathrm{~mm}$ calcein, $20 \mathrm{~mm}$ TES, $100 \mathrm{~mm} \mathrm{NaCl}, \mathrm{pH} 7.4$ ) was added to each sample. Liposomes were prepared via 10 freeze-thaw cycles in liquid nitrogen followed by incubation in a water bath at $50{ }^{\circ} \mathrm{C}$. The suspensions were then extruded 10 times through 200-nm polycarbonate membranes. After extrusion, the calcein-entrapped vesicles were separated from free calcein via gel filtration through a Sephadex G-50 column (Beijing, China) and eluted using TES buffer. The calcein-entrapped liposomes ( $90 \mu \mathrm{m}$ final concentration) were used for leakage measurements.

\section{Leakage of calcein from liposomes}

To examine the interactions of Trp-substituted AMPs with model membranes, we measured the ability of the peptides to induce calcein leakage from three types of liposomes. Liposomes containing entrapped calcein were incubated with various concentrations of peptides $(0.78-25 \mu \mathrm{M})$ for $30 \mathrm{~min}$. The fluorescence intensity of the calcein released from the liposomes was recorded at $530 \mathrm{~nm}$ with an excitation wavelength of $493 \mathrm{~nm}$. Complete $(100 \%)$ release of calcein was induced by the addition of $0.1 \%$ Triton X-100. All experiments were conducted at $25^{\circ} \mathrm{C}$, and measurements were repeated three times under 
each condition. The percentage of released calcein caused by the peptides was calculated using the following eq: ${ }^{23}$ Release $(\%)=100 \times\left(F-F_{0}\right) /\left(F_{\mathrm{t}}-F_{0}\right)$, where $F$ and $F_{\mathrm{t}}$ are the fluorescence intensities before and after the addition of Triton X-100, respectively, and $F_{0}$ is the fluorescence intensity of the intact liposomes.

\section{Scanning electron microscopy}

Scanning electron microscopry (SEM) was used to study the possible mechanism of action of Trp-substituted AMPs. Aliquots of E. coli (AS 1.349) or S. aureus (AS 1.72) $\left(\sim 3 \times 10^{7} \mathrm{CFU} \mathrm{ml}^{-1}\right)$ were incubated with each peptide $\left(1 \times\right.$ MIC) at $37^{\circ} \mathrm{C}$ for $1 \mathrm{~h}$. The bacteria were then pelleted by centrifugation at $3000 \mathrm{~g}$ for $5 \mathrm{~min}$ and twice in phosphate-buffered saline (1.5 mM KH $\mathrm{KH}_{4}, 2.7 \mathrm{~mm} \mathrm{KCl}, 8.1 \mathrm{~mm} \mathrm{Na}_{2} \mathrm{HPO}_{4}, 150 \mathrm{~mm} \mathrm{NaCl}$, pH 7.2). After removing the supernatants, the pellets were fixed with $2.5 \%$ buffered glutaraldehyde for $2 \mathrm{~h}$ at $4{ }^{\circ} \mathrm{C}$ in the dark. The bacteria were postfixed in $1 \%$ buffered osmium tetroxide for $2 \mathrm{~h}$ and then dehydrated in a sequential 30,50, $70,80,90$ and $100 \%$ ethanol series. After lyophilization and vacuum sputtercoating with gold, the samples were examined under a SEM (Hitachi SU8000, Tokyo, Japan). The tested bacterial treated without peptides as the negative control, and that treated with $\mathrm{L}-\mathrm{K} 6(1 \times \mathrm{MIC})$ as the positive control was shown for comparison.

\section{Trp fluorescence and acrylamide/KI quenching assays}

The fluorescence emission spectra of the Trp residues were determined using Varioskan Flash (Thermo Scientific, Beijing, China). Spectroscopic titration of the peptides with liposomes was performed as described by Park et al. ${ }^{24}$ Each of peptide was added at a final concentration of $10 \mu \mathrm{M}$ to the appropriate liposomes ( $5 \mathrm{~mm}$ ) in $5 \mathrm{~mm}$ TES buffer ( $\mathrm{pH}$ 7.4). The peptides and liposomes (molar ratio 1:0 to 1:100) were then allowed to equilibrate for $10 \mathrm{~min}$ at $25^{\circ} \mathrm{C}$. The fluorescence was then measured at an excitation wavelength of $280 \mathrm{~nm}$ and the emission wavelength at $300-400 \mathrm{~nm}$.

Fluorescence quenching experiments were conducted via titration of the peptides with a water-soluble quencher (either acrylamide or KI) in the absence or presence of liposomes. ${ }^{24}$ The concentration of quencher in the well ranged from 20 to $140 \mathrm{~mm}$, and the lipid/peptide molar ratio was 100:1 or 0:1. To reduce absorbance by acrylamide, excitation of tryptophan at $295 \mathrm{~nm}$ instead of $280 \mathrm{~nm}$ was used, ${ }^{25}$ and the emission was recorded at $350 \mathrm{~nm}$. The effect of the quencher on the fluorescence of each peptide was analyzed using the Stern-Volmer eq: $F_{0} / F=1+K_{\mathrm{sv}}[\mathrm{Q}]$, where $F_{0}$ and $F$ are the fluorescence intensities in the presence or absence of the quencher, respectively, $K_{\mathrm{sv}}$ is the Stern-Volmer quenching constant and $[Q]$ is the concentration of the quencher.

\section{RESULTS}

Peptide design and characterization

To investigate the role of the Trp residues in Trp-containing peptides and to improve the activity of L-K6, we designed two Trp-substituted AMPs (I1WL5W and I4WL5W) by replacing Ile or Leu residues with two Trp residues at different positions in L-K6. The amino-acid sequences, calculated molecular weights, net charges, mean hydrophobicity values $(\mathrm{H})$ and amphipathicity values of the peptides are shown in Table 1 . The mean hydrophobicity values were calculated

Table 1 Amino-acid sequences and physicochemical properties of the designed peptides

\begin{tabular}{llccc}
\hline Name & Sequence & Amphipathicity $^{\mathrm{a}}$ & $\mathrm{H}^{\mathrm{b}}$ & MW \\
\hline L-K6 & IKKILSKIKKLLK & 0.83 & 12.45 & 1663.0 \\
IIWL5W & WKKIWSKIKKLLK & 0.83 & 13.15 & 1698.2 \\
I4WL5W & IKKWWSKIKKLLK & 0.82 & 13.15 & 1698.2
\end{tabular}

Net charge of all peptides are +6 ; pl values are 10.7.

${ }^{a}$ Amphipathicity was determined by calculating the hydrophobic moment 27,28

bThe mean hydrophobicities $(H)$ of the peptides calculated using the hydrophobicity scales ${ }^{26}$

represented the total hydrophobicity (sum of all residue hydrophobicity indices) divided by the number of residues. using hydrophobicity scales (Trp, 32.4; Phe, 29.1; Leu, 23.3; Ile, 21.4; Met, 15.7; Tyr, 14.7; Val, 13.4; Pro, 9.0; Cys, 7.6; Lys, 2.8; Glu, 2.8; Ala, 2.8; Thr, 2.3; Asp, 1.6; Arg, 0.6; Gln, 0.6; His, 0; Ser, 0; Gly, 0; and Asn, -0.6). ${ }^{26}$ Amphipathicity values were calculated based on the hydrophobic moment ${ }^{27}$ using the software package, Jemboss version 1.5. ${ }^{28}$ The magnitude of the positive charge and the number of amino acids for each peptide were the same as in the L-K6 model peptide, but the $H$-values were increased from 12.45 (L-K6) to 13.15 (I1WL5W and I4WL5W). Compared with L-K6, the amphipathicity values of the two analogs were not significantly changed.

\section{Antimicrobial and hemolytic activity}

The antimicrobial and hemolytic activities of the peptides are shown in Table 2. Compared with L-K6, I1WL5W and I4WL5W, the Trp-substituted peptides of L-K6, exhibited two- to fourfold high antimicrobial activities against Gram-negative bacteria, particularly the MIC against $K$. pneumoniae, E. cloacae and P. mirabilis from $>100$ to $0.78-25 \mu \mathrm{m}$. The MIC of two peptides against Gram-positive bacteria decreased 1.5- to 4-fold at least. Neither of the peptides showed hemolytic activity at the tested concentrations $\left(\mathrm{HC}_{50}>500\right.$ $\mu \mathrm{M})$. The therapeutic index (TI) of each peptide was calculated as the ratio of the average $\mathrm{HC}_{50}$ to the MIC; thus, a larger value corresponds to a greater potency. In cases where no hemolytic activity was detectable at the highest concentration tested $(500 \mu \mathrm{M})$, an $\mathrm{HC}_{50}$ of $1000 \mu \mathrm{m}$ was used for the TI calculation. As shown in Table 2, both of the designed peptides exhibited a higher TI value than L-K6 with I4WL5W giving a threefold higher TI value than L-K6.

\section{Killing kinetics of Trp-substituted AMPs}

To further understand the bactericidal activities of I1WL5W and I4WL5W, we performed a time-killing assay against E. coli (AS 1.349) or S. aureus (AS 1.72). As shown in Figure 1, at concentrations equal to or above the MIC, bacterial counts slightly decreased within 30 min. However, I1WL5W and I4WL5W rapidly killed the bacteria

Table 2 MICs of the peptides against representative Gram-positive and Gram-negative bacteria and their hemolytic activities

\begin{tabular}{|c|c|c|c|}
\hline & \multicolumn{3}{|c|}{$M I C^{\mathrm{a}}(\mu \mathrm{M})$} \\
\hline & $L-K 6$ & $11 W L 5 W$ & $14 W L 5 W$ \\
\hline \multicolumn{4}{|l|}{$G^{-}$bacteria } \\
\hline E. coli & 3.13 & 6.25 & 3.13 \\
\hline$P$. aeruginosa & 6.25 & 6.25 & 3.13 \\
\hline K. pneumoniae & $>100$ & 25.0 & 25.0 \\
\hline E. cloacae & $>100$ & 3.13 & 0.78 \\
\hline P. mirabilis & $>100$ & 6.25 & 6.25 \\
\hline \multicolumn{4}{|l|}{$\mathrm{G}^{+}$bacteria } \\
\hline S. aureus & 5.00 & 3.13 & 1.56 \\
\hline E. faecalis & 3.13 & 1.56 & 0.78 \\
\hline E. faecium & 5.00 & 3.13 & 1.56 \\
\hline B. cereus & 6.25 & 3.13 & 3.13 \\
\hline S. epidermidis & 4.69 & 1.56 & 1.56 \\
\hline $\mathrm{HC}_{50}(\mu \mathrm{M})$ & $>500$ & $>500$ & $>500$ \\
\hline $\mathrm{Tl}^{\mathrm{b}}$ & 160.22 & 308.00 & 564.56 \\
\hline
\end{tabular}

aMIC: minimal peptide concentration required for total inhibition of microbe growth in liquid medium. The values are the means of three independent experiments performed in duplicate. bTI (Therapeutic index): The average $\mathrm{HC}_{50} / \mathrm{MIC}$ value. The percent lysis by peptides was 3.6 and $14.6 \%$ at $500 \mu \mathrm{m}$. We therefore estimated the $\mathrm{HC}_{50}$ value by linear extrapolation of the slope of the line to between 600 and $1000 \mu \mathrm{m}$. In fact, the $\mathrm{HC}_{50}$ values may be much larger. 

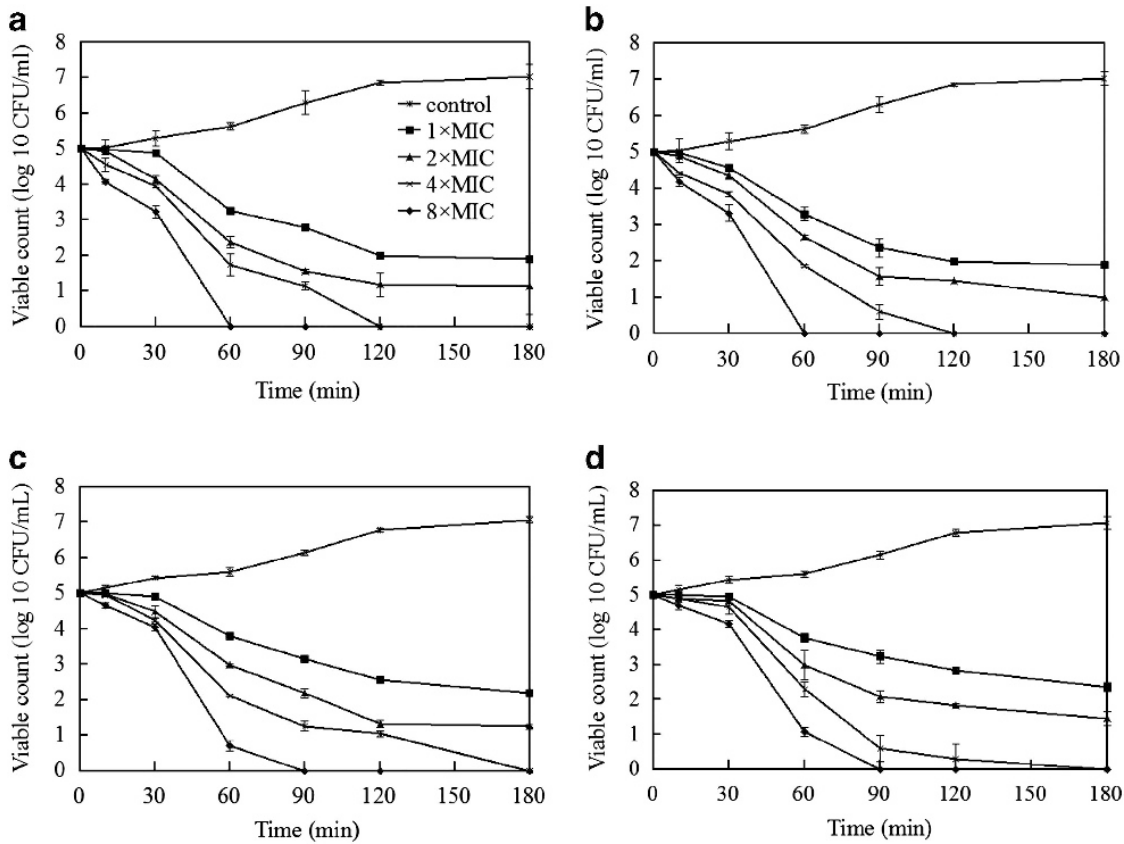

Figure 1 Time-kill curves of IIWL5W and I4WL5W. Bacteria treated with the respective peptides were diluted at the indicated times and then plated on LB agar. CFUs were counted after $16 \mathrm{~h}$ of incubation at $37^{\circ} \mathrm{C}$. I1WL5W (a and c), I4WL5W (b and d), E. coli (a and b) and $S$. aureus (c and d).
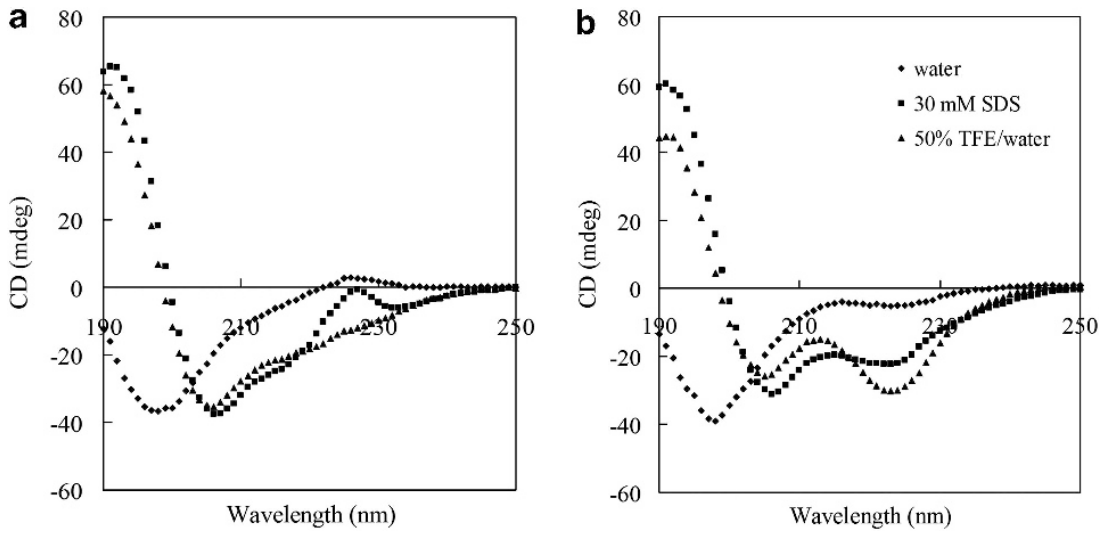

Figure 2 Circular Dichroism spectra for (a) I1WL5W and (b) I4WL5W in water, 50\% trifluoroethanol (TFE)/water and $30 \mathrm{~mm}$ SDS. Both of the peptides were unstructured in water alone, whereas they adopted an $\alpha$-helical structure in the presence of TFE or SDS micelles, conditions that mimic a membrane environment.

with a $>4 \log _{10}$ reduction in the number of viable cells only after $60 \mathrm{~min}$ at concentrations of $2 \times$ and $4 \times$ the MIC value. When the concentration equaled $8 \times$ MIC, E. coli or S. aureus were completely killed within 60 or $90 \mathrm{~min}$.

\section{CD spectra}

Using CD spectroscopy, we determined the secondary structures of I1WL5W and I4WL5W in water, $30 \mathrm{~mm}$ SDS or 50\% (v/v) TFE/water; the latter two conditions mimic the hydrophobic environment of membranes. The peptides had a random coil structure in water but adopted a highly ordered structure in $30 \mathrm{~mm}$ SDS or $50 \%$ (v/v) TFE/ water with negative double-minimum peaks at 208 and $222 \mathrm{~nm}$. These results indicated that the peptides formed an $\alpha$-helical structure (Figure 2). On the basis of the molar ellipticity at $222 \mathrm{~nm}$, I4WL5W had a higher $\alpha$-helical percentage than I1WL5W in $50 \%(\mathrm{v} / \mathrm{v})$ TFE/water or $30 \mathrm{~mm}$ SDS. In the $30 \mathrm{~mm}$ SDS solution, the calculated helical content was $41.8 \%$ and $73.3 \%$ for I1WL5W and I4WL5W, respectively, whereas in the presence of TFE, the helical content was $35.0 \%$ and $57.1 \%$ for I1WL5W and I4WL5W, respectively.

\section{Calcein leakage}

A calcein leakage assay was used to investigate the interactions between the Trp-substituted AMPs and model membranes. We determined the ability of these peptides to cause leakage of entrapped calcein from the uncharged liposomes (10:1 (w/w) PC/cholesterol) mimicked that of the human erythrocyte membrane, the negatively charged liposomes (3:1 (w/w) PG/CL or 2:1:7 (w/w/w) PG/CL/PE) mimicked those of Gram-positive ( $S$. aureus) and Gram-negative (E. coli) bacteria, respectively. As shown in Figure 3, I1WL5W and I4WL5W were associated with slight calcein leakage from the $\mathrm{PC} /$ cholesterol liposomes at the highest concentration $(25 \mu \mathrm{M})$, whereas significant leakage was observed from PG/PE/CL $(2: 7: 1$, w/w/w) 

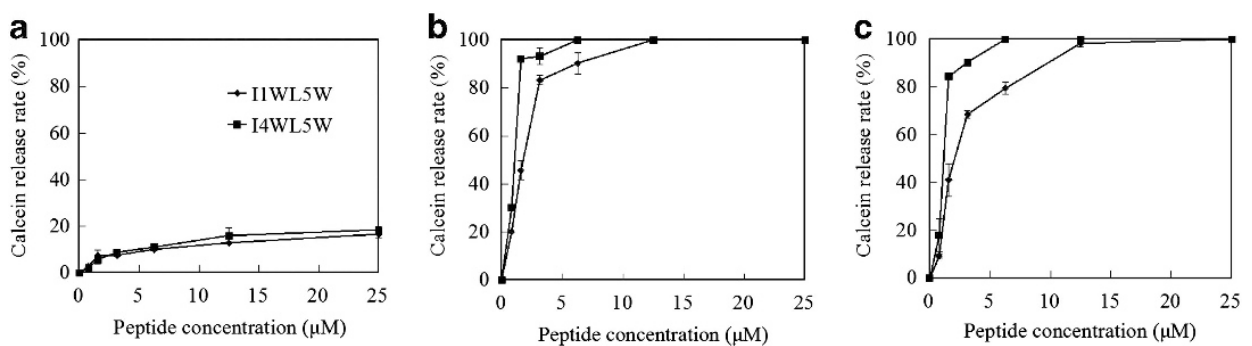

Figure 3 The release of calcein from liposomes composed of (a) phosphatidylcholine (PC)/cholesterol (10:1, w/w), (b) phosphatidylglycerol (PG)/cholesterol and cardiolipin (CL) $(3: 1, \mathrm{w} / \mathrm{w})$ and (c) PG/phosphatidylethanolamine (PE)/CL $(2: 7: 1, \mathrm{w} / \mathrm{w} / \mathrm{w})$. The fluorescence intensity is shown as a function of time after adding various concentrations of the tested peptides. The lipid concentration was $90 \mu \mathrm{m}$. Each data point represents the average of four independent experiments.
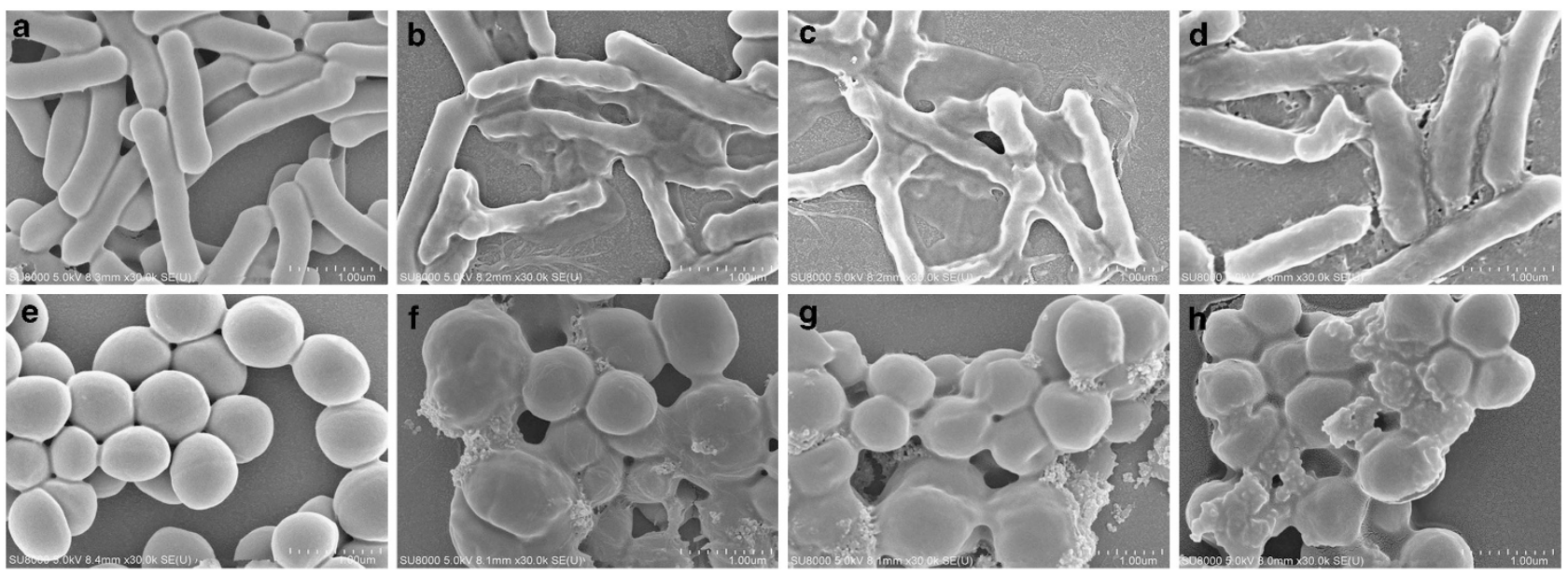

Figure 4 Scanning electron microscopy (SEM) analysis of the Trp-substituted peptides treated with bacteria. (a and e) control E. coli and S. aureus; (b-d) I1WL5W-, I4WL5W- and L-K6-treated E. coli; (f-h) I1WL5W-, I4WL5W- and L-K6-treated S. aureus. The concentration of tested peptides treated with E. coli was $6.25,3.13$ and $3.13 \mu \mathrm{m}$ (IIWL5W, I4WL5W and L-K6, respectively), and that treated with S. aureus was $3.13,1.56$ and $5.00 \mu \mathrm{m}$ (IIWL5W, I4WL5W and L-K6, respectively).

or PG/CL (3:1, w/w) liposomes treated with either peptide. Moreover, only a low concentration of peptide was required to induce high amounts of leakage. In the PG/PE/CL liposomes, the calcein leakage rate caused by I1WL5W was $79.6 \%$ at a concentration of $6.25 \mu \mathrm{M}$, whereas I4WL5W caused 100\% leakage at the same concentration. In the PG/CL liposomes, I1WL5W and I4WL5W caused $100 \%$ leakage at concentrations of $12.5 \mu \mathrm{M}$ and $6.25 \mu \mathrm{M}$, respectively. The ability of I1WL5W to cause leakage was weaker than that of I4WL5W in $\mathrm{PG} / \mathrm{PE} / \mathrm{CL}$ or PG/CL liposomes.

\section{Scanning electron microscopy}

To examine the activity of Trp-substituted AMPs against E. coli (AS 1.349) or S. aureus (AS 1.72) in more detail, we used SEM to observe the morphological changes that occurred upon incubation of the peptides with bacteria. A negative control (absence of peptides) is shown for comparison, in addition, the bacteria treated with L-K6 as a positive control. In the absence of peptides, E. coli and S. aureus had a smooth bright surface (Figures $4 \mathrm{a}$ and e). Exposure of the bacteria to a $1 \times$ MIC of I1WL5W or I4WL5W or L-K6 for 60 min resulted in a marked change in the appearance of the cells. Compared with the negative control-treated groups, the peptide-treated E. coli cells showed a marked structural change. The cells were distorted, and blebbing and cell debris were observed (Figures $4 \mathrm{~b}-\mathrm{d}$ ). The membranes of $S$. aureus also showed some blebbing and roughening (Figures $4 \mathrm{f}-\mathrm{h}$ ).
Tryptophan emission fluorescence and fluorescence quenching Tryptophan emission fluorescence by the Trp-substituted peptides was used to examine the interaction of the Trp residues with a membrane in a variety of liposomes mimicked the membrane of human erythrocyte (10:1 (w/w) PC/cholesterol), S. aureus $(3: 1(\mathrm{w} / \mathrm{w})$ and E. coli (2:7:1 (w/w/w) PG/PE/CL), respectively. A blue shift in the maximum emission wavelength occurs when a Trp residue moves into a more hydrophobic environment, and the process is typically interpreted as the indole moiety inserting into the hydrophobic core of the bilayer. In buffer, I1WL5W and I4WL5W had a Trp emission maximum at $\sim 340 \mathrm{~nm}$ (Table 3$)$. In the presence of PG/PE/CL (2:7:1, $\mathrm{w} / \mathrm{w} / \mathrm{w})$ or PG/CL $(3: 1, \mathrm{w} / \mathrm{w})$ liposomes, the blue shifts of I1WL5W (or I4WL5W) in the Trp emission spectra were maximally shifted by $16 \mathrm{~nm}$ (or $20 \mathrm{~nm}$ ) and $22 \mathrm{~nm}$ (or $20 \mathrm{~nm}$ ). However, when mixed with the $\mathrm{PC} /$ cholesterol $(10: 1, \mathrm{w} / \mathrm{w})$ liposomes, none of the peptides demonstrated a significant blue shift (Figure 5). Compared with I1WL5W, I4WL5W exhibited a larger blue shift, indicating that its Trp side chains preferentially penetrated into more rigid and hydrophobic environments within the PG/PE/CL or PG/CL liposomes.

The ability of Trp residues to access the lipid bilayer was evaluated by measuring the Stern-Volmer quenching constants $\left(K_{\mathrm{sv}}\right)$ using soluble acrylamide or KI as a quencher. If Trp residues insert into the hydrophobic core of the bilayer, they are no longer susceptible to the effects of the soluble quencher. Therefore, a low $K_{\text {sv }}$ indicates that the fluorophore is buried in the hydrophobic core of a bilayer. 
The $K_{\mathrm{sv}}$ values for the peptides are shown in Table 3 . The fluorescence of Trp decreased in a concentration-dependent manner upon the addition of either acrylamide or KI to the peptide solution in the presence of liposomes. I1WL5W and I4WL5W had much lower $K_{\mathrm{sv}}$ values in liposomes than in aqueous buffer solutions. The $K_{\mathrm{sv}}$ values for I1WL5W and I4WL5W were $\sim 42.3$ (38.9) and 50.5 (38.3), respectively, in TES buffer when the peptides were quenched by acrylamide (KI). The $K_{\mathrm{sv}}$ value for the peptides was lower in the presence of PG/PE/CL $(2: 7: 1, \mathrm{w} / \mathrm{w} / \mathrm{w})$ or PG/CL $(3: 1, \mathrm{w} / \mathrm{w})$ liposomes than in the presence of $\mathrm{PC} /$ cholesterol (10:1, w/w) liposomes, indicating that the Trp residues of the AMPs were protected in the PG/PE/CL $(2: 7: 1, w / w / w)$ or PG/CL $(3: 1, w / w)$ liposomes (Table 3; Figure 6). These data correlated well with the potent antimicrobial activities of I1WL5W and I4WL5W.

\section{DISCUSSION}

To meet the challenge of antibiotic resistance worldwide, a new generation of antimicrobials must be developed. Natural AMPs exhibit a variety of three-dimensional structures and can be used as templates for engineering a new generation of antimicrobials. One of the many strategies for peptide design is to optimize natural templates via truncation or substitution. In this study, we selected L-K6, a temporin-1CEb-derived peptide from the skin secretions of Chinese brown frog as the template for designing a series of Trp-containing peptides that possessed better antimicrobial activity. Trp residues, which contain bulkier side chains, can interact more strongly with the

Table 3 Tryptophan emission maxima of peptides and $K_{\mathrm{SV}}$ in TES (pH 7.4) or in the presence of PC/cholesterol (10:1, w/w), PG/CL (3:1, w/w) or PG/PE/CL (2:7:1, w/w/w) liposomes

\begin{tabular}{|c|c|c|c|c|c|}
\hline \multirow[b]{2}{*}{ Peptides } & \multirow[b]{2}{*}{$\begin{array}{c}\lambda_{\max } \text { buffer } \\
(\mathrm{nm})\end{array}$} & \multicolumn{4}{|c|}{$\mathrm{K}_{S V}\left(M^{-1}\right)$ (quenched by acrylamide/KI) } \\
\hline & & Buffer & $\begin{array}{c}\text { PC/cholesterol } \\
(10: 1)\end{array}$ & $\begin{array}{c}P G / C L \\
(3: 1)\end{array}$ & $\begin{array}{c}P G / P E / C L \\
(2: 7: 1)\end{array}$ \\
\hline I1WL5W & 344 & $42.3 / 38.9$ & $9.5 / 10.6$ & $5.5 / 1.2$ & 7.6/3.2 \\
\hline I4WL5W & 340 & $50.5 / 38.3$ & $7.7 / 9.1$ & 2.8/0.6 & $4.6 / 2.3$ \\
\hline
\end{tabular}

Abbreviations: $\mathrm{CL}$, cholesterol and cardiolipin; $K_{\mathrm{SV}}$, Stern-Volmer constant; PC phosphatidylcholine; $\mathrm{PE}$, phosphatidylethanolamine; PG, phosphatidylglycerol; TES, N-Tris (hydroxymethyl) methyl-2-aminoethanesulfonic acid.

$K_{\mathrm{S} v}$ values $\left(\mathrm{M}^{-1}\right)$ were determined from the Stern-Volmer eq: $F_{0} / F=1+K_{\mathrm{Sv}}[\mathrm{Q}]$, where $Q$ is the concentration of the quencher (acrylamide or $\mathrm{KI}$ ). The concentration of the quencher varied from 0.02 to $0.14 \mathrm{~m}$.

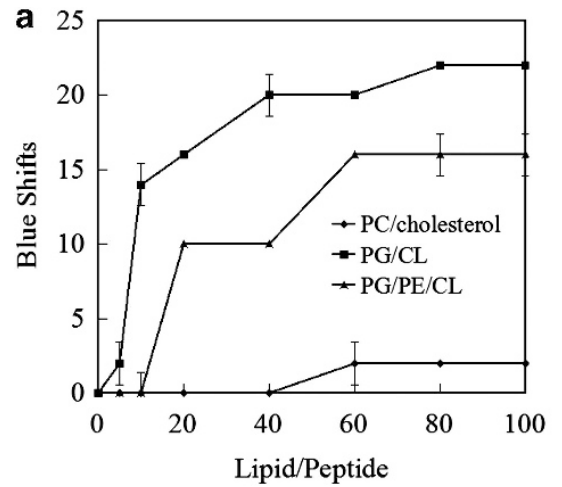

hydrophobic membrane core than other non-polar amino-acid residues, such as Tyr and Phe. ${ }^{29,30}$ In our previous study, nine Trp-containing peptides were designed by substituting Trp residues into L-K6 at different positions (Ile, ${ }^{1} \mathrm{Ile},{ }^{4} \mathrm{Leu},{ }^{5} \mathrm{Leu}^{11}$ and $\mathrm{Leu}^{12}$ ) to investigate the effects of $\operatorname{Trp}$ residues at different positions on antimicrobial activity. ${ }^{15}$ In the current study, to further improve the antimicrobial activities of Trp-containing peptides against Gramnegative and Gram-positive bacteria and to identify their interaction with the lipid bilayer, we designed peptides I1WL5W and 14WL5W containing two Trp residues at the $1 / 4$ and 5 positions, respectively. The results of the antimicrobial assay demonstrated that I1WL5W and I4WL5W had more significant activity against all of the bacterial strains tested than L-K6 or its nine Trp-substituted analogs. In particular, I1WL5W and I4WL5W exhibited higher antimicrobial activity against both Gram-positive and Gram-negative bacteria than L5WL11W, L5WL12W and L11WL12W. ${ }^{15}$ The result implies a very important role of Trp residues at positions 1,4 and 5 in the antimicrobial activity of L-K6 analogs. Compared with I1W, I4W or L5W, I1WL5W and I4WL5W also had significantly higher activity against the tested bacterial strains, suggesting that the number of Trp residues is important for antimicrobial activities. The killing kinetics against $E$. coil or $S$. aureus showed that, similar to the most cationic AMPs, I1WL5W and I4WL5W killed Gram-positive and Gramnegative bacteria rather than inhibiting their growth. I1WL5W and I4WL5W can completely kill $E$. coli in $60 \mathrm{~min}$ at $8 \times$ MIC and in $120 \mathrm{~min}$ at $4 \times \mathrm{MIC}$, and completely kill $S$. aureus in $90 \mathrm{~min}$ at $8 \times$ MIC and in $180 \mathrm{~min}$ at $4 \times$ MIC, suggesting the bactericidal activity of I1WL5W and I4WL5W at high concentration compared with the present antibiotics. Both I1WL5W and I4WL5W induced slight hemolysis at a concentration of $500 \mu \mathrm{M}$, causing only $3.6 \%$ and $14.6 \%$ hemolysis, respectively.

Linear AMPs are classified according to their primary structure. However, most AMPs (regardless of their length or amino-acid sequence) form an amphipathic $\alpha$-helix when bound to lipid bilayers with their polar and apolar amino acids on opposing surfaces along the long axis of the helix. ${ }^{31,32}$ In our study, the results of CD spectra showed that I1WL5W and I4WL5W largely adopted an amphipathic $\alpha$-helical structure in the membrane environment, but were relatively unstructured in aqueous solution. The $\alpha$-helix formation of a peptide is closely related to its interaction with the hydrophobic core of a bilayer, and the structure has an important role in the antimicrobial activity of AMPs. ${ }^{24,28}$ I1WL5W and I4WL5W adopted a $35-43 \%$ and a $57-73 \% \alpha$-helical conformation in the $50 \%$ TFE and $30 \mathrm{~mm}$ SDS

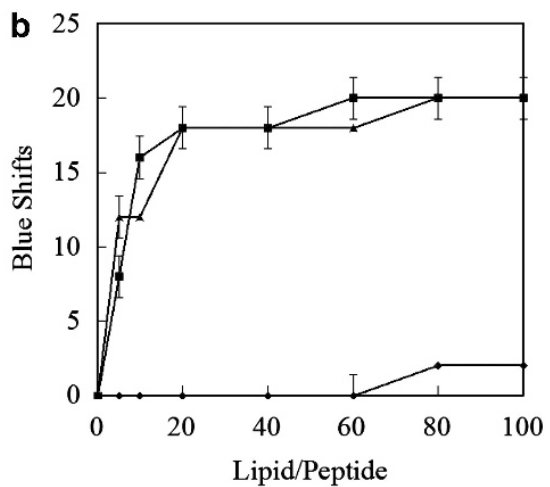

Figure 5 Blue shifts in Trp emission maxima of I1WL5W (a) and I4WL5W (b) in phosphatidylcholine (PC)/cholesterol (10:1, w/W), phosphatidylglycerol $(\mathrm{PG}) /$ cholesterol and cardiolipin (CL) $(3: 1, \mathrm{w} / \mathrm{w})$ and PG/phosphatidylethanolamine (PE)/CL (2:7:1, w/w/w) liposomes. Each data point is the average of four independent experiments \pm s.e.m. 

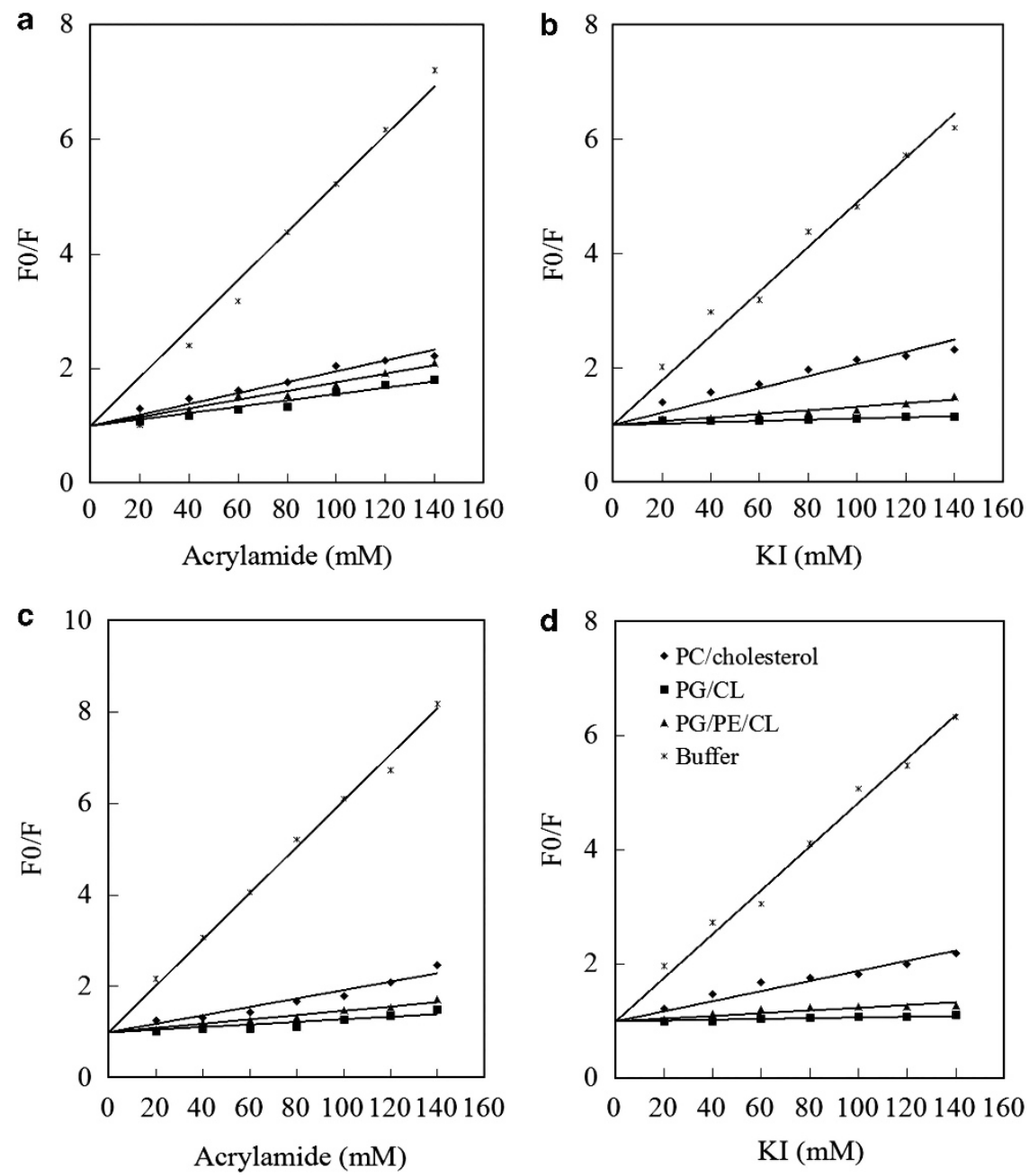

Figure 6 The Stern-Volmer plots for the quenching of the fluorescence of the Trp residue of IIWL5W (a and $\mathbf{b}$ ) or I4WL5W (c and $\mathbf{d}$ ) by acrylamide/KI in an aqueous buffer or in the presence of phosphatidylcholine (PC)/cholesterol (10:1, w/w), phosphatidylglycerol (PG)/cholesterol and cardiolipin (CL) (3:1, w/w) and $\mathrm{PG} /$ phosphatidylethanolamine (PE)/CL $(2: 7: 1, \mathrm{w} / \mathrm{w} / \mathrm{w})$ liposomes.

solutions, respectively. In addition, the TI value of I4WL5W was onefold greater than that of I1WL5W. Interestingly, the secondary structure predicted by the helical wheel model using ANTHEPROT 4.3 (Institute of Biology and Chemistry of Proteins, France) showed that the Trp residue at positions 1 and 4 was located at the center of a hydrophobic cluster (data not shown), but I1WL5W had a significantly lower $\alpha$-helical content than I4WL5W.

Previous studies have shown that the mechanism of action of AMPs involves two stages, initial electrostatic interaction of the cationic residues of the peptides with the anionic lipids of the bacterial membrane $e^{33,34}$ followed by hydrophobic interaction of aromatic side chains-containing amino acids with the lipid bilayer. Consistent with these results, the $\operatorname{Trp}$ blue shifts and $K_{\mathrm{sv}}$ values indicated that I1WL5W and I4WL5W inserted Trp residues into the lipid bilayer of PG/PE/CL $(2: 7: 1, \mathrm{w} / \mathrm{w} / \mathrm{w})$ or PG/CL $(3: 1, \mathrm{w} / \mathrm{w})$ liposomes, mimicked the membrane of E. coli or $S$. aureus and induced calcein leakage. The Trp-substituted AMPs bound more tightly to bacterial membrane-mimicking liposomes than to those mimicking a mammalian membrane, suggesting that anionic lipids promote the initial electrostatic interaction between positively charged AMPs and the negatively charged membrane. Because the mammalian membrane is deficient in anionic lipids, AMPs cannot undergo a proper initial interaction. As previously reported, it is the hydrophobic interaction between AMPs and the lipid bilayer core that is essential for membrane disruption., ${ }^{9,17,35}$ In addition, SEM analysis suggested that the peptides interacted with and disrupted the cytoplasmic membrane of the bacteria, leading to dissolution of the membrane and cell death. The results also indicated that the Trpsubstituted AMPs killed the bacteria specifically by disrupting the bacterial cell membrane. Thus, Trp residues contributed to the insertion of the Trp-substituted AMPs into the lipid bilayers of bacterial membranes, thereby disrupting the membranes more efficiently. Trp-substituted AMPs offer potential as new drug candidates because they exhibit improved antimicrobial activity against Gram-positive and Gram-negative bacteria compared with the model peptide.

\section{ACKNOWLEDGEMENTS}

This work was supported by the National Natural Science Foundation of China (grant no. 31272314 and no. 81202448), the Natural Science Foundation of Liaoning (grant no. 201202121) and the Program for Liaoning Innovative Research Team in University (LT2012019). 
1 Hancock, R. E. Cationic peptides: effectors in innate immunity and novel antimicrobials. Lancet Infect. Dis. 1, 156-164 (2001).

2 Brogden, N. K. \& Brogden, K. A. Will new generations of modified antimicrobia peptides improve their potential as pharmaceuticals? Int. J. Antimicrob. Agents 38, 217-225 (2011).

3 Fjell, C. D., Hiss, J. A., Hancock, R. E. \& Schneider, G. Designing antimicrobial peptides: form follows function. Nat. Rev. Drug Discov. 11, 37-51 (2011).

4 Giuliani, A. \& Rinaldi, A. C. Beyond natural antimicrobial peptides: multimeric peptides and other peptidomimetic approaches. Cell Mol. Life Sci. 68, 2255-2266 (2011)

5 Klüver, E., Adermann, K. \& Schulz, A. Synthesis and structure-activity relationship of $\beta$-defensins, multi-functional peptides of the immune system. J. Pept. Sci. 12, 243-257 (2006).

6 Koo, Y. S. et al. Structure-activity relations of parasin I, a histone H2A-derived antimicrobial peptide. Peptides 29, 1102-1108 (2008).

7 Saberwal, G. \& Nagaraj, R. Cell-lytic and antibacterial peptides that act by perturbing the barrier function of membranes: facets of their conformational features, structurefunction correlations and membrane perturbing activities. Biochim. Biophys. Acta 1197, 109-131 (1994).

8 Epand, R. F. et al. Direct comparison of membrane interactions of model peptides composed of only Leu and Lys residues. Biopolymers 71, 2-16 (2003).

9 Kiyota, T., Lee, S. \& Sugihara, G. Design and synthesis of amphiphilic a-helical model peptides with systematically varied hydrophobic-hydrophilic balance and their interaction with lipidand bio-membranes. Biochemistry 35, 13196-13204 (1996).

10 Song, Y. M. et al. Cell selectivity and mechanism of action of antimicrobial model peptides containing peptoid residues. Biochemistry 44, 12094-12106 (2005).

11 Khandelia, H. \& Kaznessis, Y. N. Cation- $\pi$ interactions stabilize the structure of the antimicrobial peptide indolicidin near membranes: moleculardynamics simulations. J. Phys. Chem. B 111, 242-250 (2007).

12 Shepherd, C. M., Vogel, H. J. \& Tieleman, D. P. Interactions of the designed antimicrobial peptide MB21 and truncated dermaseptin S3 with lipid bilayers: molecular-dynamics simulations. Biochem. J. 370, 233-243 (2003).

13 Aliste, M. P., MacCallum, J. L. \& Tieleman, D. P. Molecular dynamics simulations of pentapeptides at interfaces: salt bridge and cation-pi interactions. Biochemistry $\mathbf{4 2}$ 8976-8987 (2003).

14 Yau, W. M., Wimley, W. C., Gawrisch, K. \& White, S. H. The preference of tryptophan for membrane interfaces. Biochemistry 37, 14713-14718 (1998).

$15 \mathrm{Bi}$, X., Wang, C., Ma, L., Sun, Y. \& Shang, D. Investigation of the role of tryptophan residues in cationic antimicrobial peptides to determine the mechanism of antimicrobial action. J. Appl. Microbiol. 115, 663-672 (2013).

16 Pál, T., Abraham, B., Sonnevend, A., Jumaa, P. \& Conlon, J. M. Brevinin1BYa: a naturally occurring peptide from frog skin with broad-spectrum antibacterial and antifungal properties. Int. J. Antimicrob. Agents 27, 525-529 (2006).

17 Lequin, O. et al. Dermaseptin S9, an alpha-helical antimicrobial peptide with a hydrophobic core and cationic termini. Biochemistry 45, 468-480 (2006).

18 Kim, J. Y., Park, S. C., Yoon, M. Y., Hahm, K. S. \& Park, Y. C-terminal amidation of PMAP-23: translocation to the inner membrane of Gram-negative bacteria. Amino Acids 40, 183-195 (2011).
19 Chen, Y., Mant, C. T. \& Hodges, R. S. Preparative reversed-phase high-performance liquid chromatography collection efficiency for an antimicrobial peptide on columns of varying diameters ( $1 \mathrm{~mm}$ to $9.4 \mathrm{~mm}$ I.D.). J. Chromatogr. A 1140, 112-120 (2007).

20 Epand, R. F., Schmitt, M. A., Gellman, S. H. \& Epand, R. M. Role of membrane lipids in the mechanism of bacterial species selective toxicity by two a/b-antimicrobial peptides. Biochim. Biophys. Acta. 1758, 1343-1350 (2006).

21 Ishibashi, J., Saido-Sakanaka, H., Yang, J., Sagisaka, A. \& Yamakawa, M. Purification, cDNA cloning and modification of a defensin from the coconut rhinoceros beetle, Oryctes rhinoceros. Eur. J. Biochem. 266, 616-623 (1999).

22 Coccia, C. et al. Membrane interaction and antibacterial properties of two mildly cationic peptide diastereomers, bombinins $\mathrm{H} 2$ and $\mathrm{H} 4$, isolated from Bombina skin. Eur. Biophys. J. 40, 577-588 (2011).

23 Matsuzaki, K., Sugishita, K. \& Miyajima, K. Interactions of an antimicrobial peptide, magainin 2, with lipopolysaccharide containing liposomes as a model for outer membranes of Gram-negative bacteria. FEBS Lett. 449, 221-224 (1999).

24 Park, N. G. et al. Conformation of tachyplesin I from Tachypleus tridentatus when interacting with lipid matrices. Biochemistry 31, 12241-12247 (1992).

25 De Kroon, A. I., Soekarjo, M. W., De Gier, J. \& De Kruijff, B. The role of charge and hydrophobicity in peptide-lipid interaction: a comparative study based on tryptophan fluorescence measurements combined with the use of aqueous and hydrophobic quenchers. Biochemistry 29, 8229-8240 (1990).

26 Mant, C. T., Kovacs, J. M., Kim, H. M., Pollock, D. D. \& Hodges, R. S. Intrinsic amino acid side-chain hydrophilicity/hydrophobicity coefficients determined by reversed-phase high-performance liquid chromatography of model peptides: comparison with other hydrophilicity/hydrophobicity scales. Biopolymers 92, 573-595 (2009).

27 Eisenberg, D., Weiss, R. M. \& Terwilliger, T. C. The helical hydrophobic moment: measure of the amphiphilicity of a helix. Nature 299, 371-374 (1982).

28 Carver, T. \& Bleasby, A. The design of Jemboss: a graphical user interface to EMBOSS Bioinformatics 19, 1837-1843 (2003).

29 Strom, M. B. et al. Important structural features of 15-residue lactoferricin derivatives and methods for improvement of antibacterial activity. Biochem. Cell Biol. 80, 65-74 (2002).

30 Wimley, W. C. \& White, S. H. Experimentally determined hydrophobicity scale for proteins at membrane interfaces. Nat. Struct. Biol. 3, 842-848 (1996).

31 Apponyi, M. A. et al. Host-defense peptides of Australian anurans: structure mechanism of action, and evolutionary significance. Peptides 25, 1035-1054 (2004).

32 Nicolas, P., Vanhoye, D. \& Amiche, M. Molecular strategies in biological evolution of antimicrobial peptides. Peptides 24, 1669-1680 (2003).

33 Juffer, A. H., Shepherd, C. M. \& Vogel, H. J. Protein-membrane electrostatic interactions: applications of the lekner summation technique. J. Chem. Phys. 114, 1892-1905 (2001).

34 Shepherd, C. M., Schaus, K. A., Vogel, H. J. \& Juffer, A. H. Molecular dynamics study of peptide-bilayer adsorption. Biophys. J. 80, 579-596 (2001).

35 Henzler-Wildman, K. A., Martinez, G. V., Brown, M. F. \& Ramamoorthy, A. Perturbation of the hydrophobic core of lipid bilayers by the human antimicrobial peptide LL-37. Biochemistry. 43, 8459-8469 (2004). 